\title{
MIXED PERIODIC JACOBI CONTINUED FRACTIONS
}

\author{
YOSHIFUMI KATO
}

\section{$\S 1$. Introduction}

Let $b_{0}$ be a positive real number and

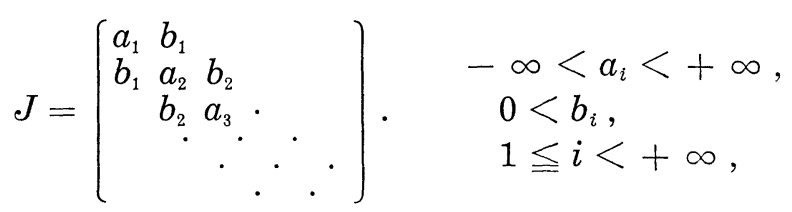

be a Jacobi matrix. We can associate with them a Jacobi continued fraction, which will be abbreviated to a $J$ fraction from the next section, as follows

$$
\begin{aligned}
\varphi(z) & =\frac{b_{0}^{2} \mid}{\mid z-a_{1}}-\frac{b_{1}^{2}}{\mid z-a_{2}}-\frac{b_{2}^{2} \mid}{\mid z-a_{3}}-\cdots \\
& =\lim _{n \rightarrow \infty} \frac{A_{n}(z)}{B_{n}(z)}
\end{aligned}
$$

where $A_{n}(z) / B_{n}(z)$ is the $n$-th Padé approximant of $\varphi(z)$. Under a suitable condition, which is always satisfied if $\max _{i}\left\{\left|a_{i}\right|,\left|b_{i}\right|\right\} \leq{ }^{\exists} M<+\infty$ holds, $\varphi(z)$ can be described in a Stieltjes transform

$$
\varphi(z)=\int_{-\infty}^{+\infty} \frac{d \mu(x)}{z-x}
$$

for some Stieltjes measure $d \mu(x)$ on the real axis. And the denominators $B_{n}(x), 0 \leq n<+\infty$, constitute a system of orthogonal polynomials with respect to $d \mu(x)$.

In the previous papers [2], [3], we investigate what kind of measures $d \mu(x)$ gives a purely periodic Jacobi continued fraction, that is, $a_{i+N}=a_{i}$, $1 \leq i<+\infty, b_{i+N}=b_{i}, 0 \leq i<+\infty$, for some positive integer $N$. In fact we succeed in showing that such a function $\varphi(z)$ can be explicitly written by means of abelian integrals on special hyperelliptic curves $\mathscr{R}$ introduced by van Moerbeke P. and Mumford D. [7]. If $N=1, B_{n}(x)$ is

Received June 11, 1985. 
essentially the $n$-th Tschebyscheff polymonial of the second type. But in case $N>1$, we find a new phenomenon related to discrete measures.

This paper is the continuation of the preceding papers and we devote ourselves to study mixed periodic Jacobi continued fractions. Namely we deal with the followings

$\begin{aligned} &M \alpha) a_{i+N}=a_{i} \text { for } i \geq M \text { and } b_{i+N}=b_{i} \text { for } i \geq M-1 \\ & \text { but } \quad a_{M-1+N} \neq a_{M-1}, \\ &M \beta) \quad a_{i+N}=a_{i}, \quad b_{i+N}=b_{i} \text { for } i \geq M \text { but } b_{M-1+N} \neq b_{M-1},\end{aligned}$

where $M$ and $N$ are positive integers. We refer them to be of type $M \alpha$ and $M \beta$ respectively. Our main results are stated in Section three. See Theorems 3.4 and 3.7. We give there complete characterizations of these functions and concrete methods to construct them. And the Stieltjes measures $d \mu(x)$ are explicitly described. Our work is closely related to physical problems. Namely some diagonal elements of the Hamiltonian of a tight binding formalism in solid physics which treat semiconductors or alloys admit a Jacobi continued fraction. And the measure $d \mu(x)$ represents the density of energy states of electrons. The non periodic parts of $M \alpha$ ) and $M \beta$ ) correspond to the effects of impurities. See, for examples, Lambin, Ph. and Gaspard, J-P. [4] and Turchi, P., Ducastelle, F. and Tréglia, G. [11].

The author would like to express his thanks to Prof. Magnus A. for giving useful comments to the problems which he proposed at the International Symposium on Orthogonal Polynomials and their Applications held in France. The Proceedings will be published as one of the series of Springer Lecture Notes in Mathematics.

\section{§2. Purely periodic $J$ fractions}

First we recall some preliminary knowledge and notation from [2] about purely periodic $J$ fractions. But we slightly change the terminology.

Let

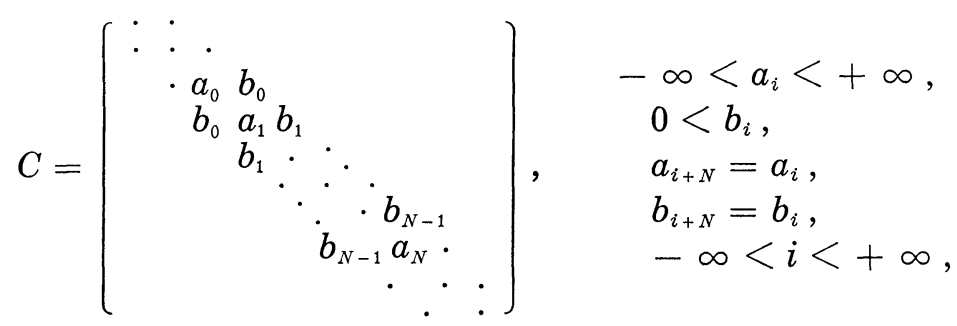


be a complete $N$ periodic Jacobi matrix and $f=\left(\cdots, f_{-1}, f_{0}, f_{1}, \cdots\right)^{t}$ be an infinite column vector. The matrix $C$ acts canonically on $f$. We denote by $D$ the shift operator of degree $+1,(D f)_{i}=f_{i+1}$ and set $S=D^{N}$. The condition that $C$ and $S$ admit a common eigenvector is parametrized by the following open Riemann surface

$$
\begin{aligned}
\mathscr{R}_{0} & =\left\{(z, h) \in C \times C^{*} \mid C f=z f, S f=h f \text { for some } f \neq 0\right\} \\
& =\left\{(z, h) \in C \times C^{*}|\operatorname{det}| z I-C_{h} \mid=F\left(h, h^{-1}, z\right)=0\right\} .
\end{aligned}
$$

We denote by $C_{h}$ the $N \times N$ matrix

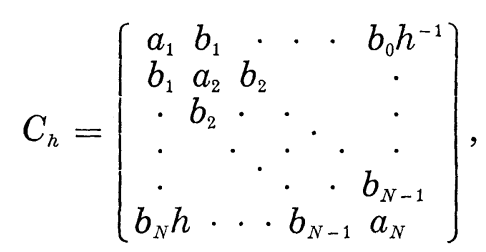

and hence

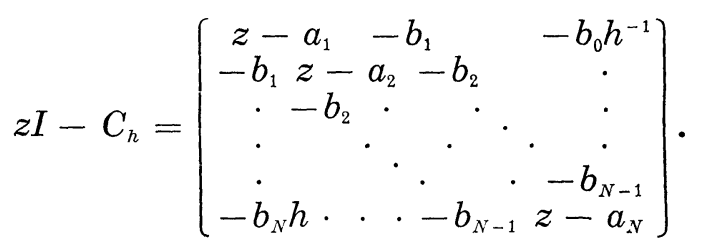

An easy calculation provides

$$
F\left(h, h^{-1}, z\right)=-A\left(h+h^{-1}\right)+P(z)
$$

where $A=\prod_{i=1}^{N} b_{i}$ and $P(z)$ is a monic polynomial of degree $N$ with real coefficients as follows

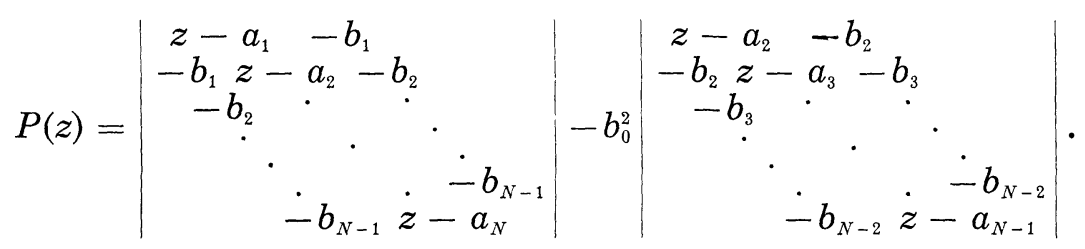

From (2.4), we have

$$
h(z)=\frac{1}{2 A}\left\{P(z) \pm \sqrt{P(z)^{2}-4 A^{2}}\right\}=\frac{1}{\frac{1}{2 A}\left\{P(z) \mp \sqrt{\left.P(z)^{2}-4 A^{2}\right\}}\right.}
$$

The expression (2.6) implies that we can easily compactify $\mathscr{R}_{0}$ by adding 
two points $P$, whose $h=\infty$, and $Q$, whose $h=0$, over $z=\infty$. The curve $\mathscr{R}=\mathscr{R}_{0} \cup\{P, Q\}$ becomes a hyperelliptic curve of genus $g=N-1$ branched at the $2 N$ zeroes $\lambda_{1}, \lambda_{2}, \cdots, \lambda_{2 N}$ of the polynomial $P(z)^{2}-4 A^{2}$. As is explained in [2], they are all real and we can arrange them in increasing order

$$
\lambda_{1}<\lambda_{2} \leq \lambda_{3}<\lambda_{4} \leq \cdots \leq \lambda_{2 N-3}<\lambda_{2 N-2} \leq \lambda_{2 N-1}<\lambda_{2 N} .
$$

The interval $\left[\lambda_{2 k-1}, \lambda_{2 k}\right], 1 \leq k \leq N$, is called the $k$-th stable band and the interval $\left[\lambda_{2 k}, \lambda_{2 k+1}\right], 1 \leq k \leq N-1$, called the $k$-th finite unstable band. The infinite interval $\left(-\infty, \lambda_{1}\right]$ (resp. $\left.\left[\lambda_{2 N},+\infty\right)\right)$ is the left (resp. right) exterior unstable band.

Remark 2.1. 1) Hereafter we denote by $\sqrt{P(z)^{2}-4 A^{2}}$ the radical of $P(z)^{2}-4 A^{2}$ which is approximately equal to $P(z)$ near the infinite point corresponding to $Q$.

2) Assume that we choose a real positive number $A$ and a monic polynomial $P(z)$ of degree $N$ with real coefficients such that the zeroes of $P(z)^{2}-4 A^{2}$ are all real. Then conversely the hyperelliptic curve $\mathscr{R}$ being defined by (2.6) comes from some complete $N$ periodic Jacobi matrix $C$, (2.1). This fact is derived from the general theory of van Moerbeke, P. and Mumford, D. [7].

We decompose $\mathscr{R}$ into three parts

$$
\mathscr{R}=\mathscr{R}_{+} \cup \mathscr{R}_{R} \cup \mathscr{R}_{-}
$$

where

$$
\begin{aligned}
& \mathscr{R}_{+}=\{p \in \mathscr{R}|| h(p) \mid>1\}, \\
& \mathscr{R}_{R}=\{p \in \mathscr{R}|| h(p) \mid=1\}, \\
& \mathscr{R}_{-}=\{p \in \mathscr{R}|| h(p) \mid<1\} .
\end{aligned}
$$

The point $P$ belongs to $\mathscr{R}_{+}$and $Q$ belongs to $\mathscr{R}_{-}$. If we project $\mathscr{R}_{+}$and $\mathscr{R}_{\text {- }}$ onto the $z$-plane, both of them become biholomorphic to the domain $\{z$-plane $\} \cup\{\infty\}-\bigcup_{k=1}^{N}\left[\lambda_{2 k-1}, \lambda_{2 k}\right]$. Therefore for any element $z_{0}$ in this domain, we can lift it up to two points $z_{0}^{+} \in \mathscr{R}_{+}$and $z_{0}^{-} \in \mathscr{R}_{-}$.

Let $f=\left(\cdots, f_{-1}, f_{0}, f_{1}, \cdots\right)^{t}$ be a common eigenvector of $C$ and $S$ under the normalization $f_{0} \equiv 1$. Then $f_{i},-\infty<i<+\infty$, become meromorphic functions on $\mathscr{R}$ and it follows that $f_{N}=h, f_{i+N}=f_{i} \cdot f_{N}=f_{i} \cdot h$. If we put $\bar{f}=\left(f_{1}, \cdots, f_{N}\right)^{t}=\left(f_{1}, \cdots, h\right)^{t}$ then $\left(z I-C_{h}\right) \bar{f}=0$. So we have 


$$
f_{i}=\frac{\Delta_{k, i}}{\Delta_{k, j}} \cdot f_{j}, \quad 1 \leq i, j, k \leq N
$$

where $\Delta_{i, j}$ denotes the $(i, j)$ cofactor of $z I-C_{h}$. The cofactor $\Delta_{N, N}$ is a monic polynomial in $z$ of degree $N-1$ whose zeroes are all real. If we arrange them in increasing order $\mu_{1}<\mu_{2}<\ldots<\mu_{N-1}$, each $\mu_{k}$ lies on the $k$-th unstable band. Hence if we denote by $\sqrt{P(x)^{2}-4 A^{2}}$ the limit

$$
\lim _{\varepsilon \downarrow 0} \sqrt{P(x+i \varepsilon)^{2}-4 A^{2}}, \quad x \in \mathscr{R}_{R}, \quad x+i \varepsilon \in \mathscr{R}_{-},
$$

the form

$$
\frac{1}{2 \pi i} \cdot \frac{\sqrt{P(x)^{2}-}-\overline{4 A^{2}}}{\Delta_{N, N}(x)} d x
$$

gives a positive measure on each stable band.

Under the same assumptions until now, we obtain the following fundamental lemmas. See the proof in [2].

Lemma 2.2. Let

$$
\begin{gathered}
\varphi(z)=\frac{b_{0}^{2}}{\mid z-a_{1}}-\frac{b_{1}^{2}}{\mid z-a_{2}}-\frac{b_{2}^{2} \mid}{\mid z-a_{3}}-\cdots \\
a_{i+N}=a_{i}, \quad 1 \leq i<+\infty, \\
b_{i+N}=b_{i}, \quad 0 \leq i<+\infty,
\end{gathered}
$$

be a purely $N$ periodic $J$ fraction. Then after an analytic prolongation, $\varphi(z)$ coincides with $b_{0} \cdot f_{1}$. The analytic prolongation is possible from the neighborhood of $Q$.

We denote the residue of $\varphi(z)$ at $\mu_{\imath}^{-} \in \mathscr{R}_{-} \subset \mathscr{R}$ by

$$
\gamma^{i}=\operatorname{Res}_{\mu_{i}}-\varphi(z)=\operatorname{Res}_{\mu_{i}^{-}} b_{0} \cdot f_{1}
$$

and put

$$
S=S(\varphi)=\left\{i \mid \gamma^{i} \neq 0\right\} \subset\{1,2, \cdots, N-1\} .
$$

And we associate with it a divisor

$$
\mathscr{D}_{S}=\sum_{i \in S} \mu_{i}^{-}+\sum_{j \in S^{c}} \mu_{j}^{+}
$$

LEMMA 2.3. We have 
1) $\gamma^{i}=0 \quad$ or $\quad-\frac{\sqrt{P\left(\mu_{i}^{-}\right)^{2}-4 A^{2}}}{\prod_{j \neq i}\left(\mu_{i}-\mu_{j}\right)}>0$.

2) From (2.10), (2.14), by putting

$$
d \mu(x)=d \mu_{S, d}(x)+d \mu_{c}(x)
$$

where

$$
\begin{aligned}
& d \mu_{S, d}(x)=\sum_{i \in S} \gamma^{i} \delta\left(x-\mu_{i}\right) d x, \quad \text { discrete measure, } \\
& d \mu_{c}(x)=\sum_{j=1}^{N} \frac{1}{2 \pi i} \chi_{\left[\lambda_{2 j-1}, \lambda_{2}\right]}(x) \frac{\sqrt{P(x)^{2}-4 A^{2}}}{\Delta_{N, N}(x)} d x \\
& \text { continuous measure },
\end{aligned}
$$

we obtain a Stieltjes measure. Then $\varphi(z)$ is the Stieltjes transform of it

$$
\varphi(z)=\int_{-\infty}^{+\infty} \frac{d \mu(x)}{z-x} .
$$

Lemma 2.4. The following three conditions are all equivalent up to a nonzero constant factor.

1) $\varphi(z)$ admits a purely $N$ periodic $J$ fraction expansion.

2) $\varphi(z)$ is the first component $f_{1}$ of the common eigenvectors of $C$ and $S$.

3) $\varphi(z)$ belongs to $L\left(\mathscr{D}_{S}+P-Q\right)$.

For any other subset $S^{\prime} \subset\{1,2, \cdots, N-1\}$, there corresponds another purely $N$ periodic $J$ fraction. We can calculate it from $\varphi(z)$ by a standard method. See Appendix. Hereafter when we want to emphasize the dependence of $\varphi(z)$ on $S$, we denote it by $\varphi_{S}(z)$.

By use of Lemma 2.2 and (2.8), we can rewrite $\varphi(z)=\varphi_{S}(z)$ as follows

$$
\begin{aligned}
& \varphi(z)=\frac{b_{0}^{2} \mid}{\mid z-a_{1}}-\frac{b_{1}^{2}}{\mid z-a_{2}}-\frac{b_{2}^{2} \mid}{\mid z-a_{3}}-\cdots \\
& =b_{0} f_{1} \\
& =\frac{A h+b_{0}^{2} \cdot\left|\begin{array}{c}
z-a_{2}-b_{2} \\
-b_{2} z-a_{3}-b_{3} \\
-b_{3} \cdot \cdot \cdot \cdot \\
\cdot \cdot \cdot \cdot b_{N-2} \\
\frac{-b_{N-2}}{z-a_{N-1}}
\end{array}\right|}{\Delta_{N, N}(x)} \\
& =\frac{1}{2} \frac{\Lambda_{0}(z)-\sqrt{P(z)^{2}-4 A^{2}}}{\Gamma_{0}(z)}
\end{aligned}
$$


where we put

$$
\begin{aligned}
& \Gamma_{0}(z)=\Delta_{N, N}(z),
\end{aligned}
$$

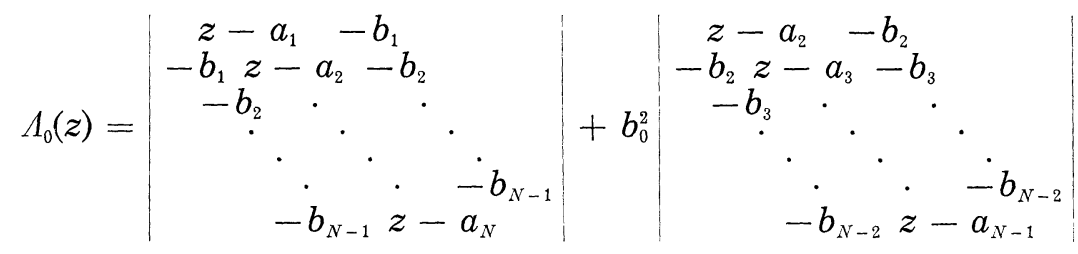

$$
\begin{aligned}
& =z^{N}+c z^{N-1}+\cdots \text {. }
\end{aligned}
$$

Here $c=a_{1}+a_{2}+\cdots+a_{N}$. Notice that $c$ coincides with the coefficient of $z^{N-1}$ in $P(z)$, so it is fixed by the hyperelliptic curve $\mathscr{R}$ and is independent of $\mu_{i}, 1 \leq i \leq N-1$. If we compare Lemma 2.4. 3) and (2.19), we have

$$
\Lambda_{0}\left(\mu_{i}\right)= \begin{cases}-\sqrt{P\left(\mu_{i}^{-}\right)^{2}-4 A^{2}} & \text { if } i \in S \\ +\sqrt{P\left(\mu_{i}^{-}\right)^{2}-4 A^{2}} & \text { if } i \in S^{c} .\end{cases}
$$

From (2.21), (2.22), $\Lambda_{0}(z)$ is uniquely determined by use of Lagrange's interpolation. The importance of the following fact is pointed out by Prof. A. Magnus.

Corollary 2.5. $\Gamma_{0}(z)$ divides the polynomial $\Lambda_{0}(z)^{2}-P(z)^{2}+4 A^{2}$.

\section{$\S 3$. Mixed periodic $J$ fractions}

In this section we study mixed periodic $J$ fractions. We represent them as follows

$$
\begin{aligned}
\psi_{M}(z)= & \frac{B_{M}^{2}}{\mid z-A_{M-1}}-\frac{B_{M-1}^{2}}{\mid z-A_{M-2}}-\cdots-\frac{B_{1}^{2}}{\mid z-A_{0}}-\frac{B_{0}^{2}}{\mid z-a_{1}} \\
& -\frac{b_{1}^{2} \mid}{\mid z-a_{2}}-\frac{b_{2}^{2} \mid}{\mid z-a_{3}} \cdots
\end{aligned}
$$

where $a_{i+N}=a_{i}, b_{i+N}=b_{i}, 1 \leq i<+\infty$. According to the number $B_{0}$, we distinguish them into two types

$$
\begin{array}{ll}
M \alpha) \quad B_{0}=b_{0} \text { but } A_{0} \neq a_{N}, \\
M \beta) \quad B_{0} \neq b_{0} .
\end{array}
$$

If we denote the purely $N$ periodic part by 


$$
\varphi(z)=\varphi_{S}(z)=\frac{b_{0}^{2}}{\mid z-a_{1}}-\frac{b_{1}^{2} \mid}{\mid z-a_{2}}-\frac{b_{2}^{2} \mid}{\mid z-a_{3}}-\cdots,
$$

we can write

$$
\psi_{M}(z)=\frac{B_{M}^{2}}{\mid z-A_{M-1}}-\frac{B_{M-1}^{2} \mid}{z-A_{M-2}}-\cdots-\frac{B_{1}^{2}}{\mid z-A_{0}-\left(\frac{B_{0}}{b_{0}}\right)^{2} \varphi(z)} .
$$

For $1 \leq l \leq M-1$, we use the notation

$$
\psi_{M}(z)=\frac{B_{M}^{2} \mid}{\mid z-A_{M-1}}-\frac{B_{M-1}^{2} \mid}{\mid z-A_{M-2}}-\cdots-\frac{B_{M-l-1}^{2}}{\mid z-A_{M-l}-\psi_{M-l}(z)} .
$$

From the expression (3.5), $\psi_{M}(z)$ is also a meromorphic function on the hyperelliptic curve $\mathscr{R}$ and the $J$ fraction expansion is significant in the neighborhood of $Q \in \mathscr{R}$.

Let $\Gamma(z)$ be a monic polynomial with real coefficients and decompose it into

$$
\begin{gathered}
\Gamma(z)=\prod_{i=1}^{r}\left(z-\nu_{i}\right) \prod_{j=1}^{s}\left(z-\zeta_{j}\right) \prod_{j=1}^{s}\left(z-\bar{\zeta}_{j}\right), \\
r+2 s=\operatorname{deg} \Gamma(z) .
\end{gathered}
$$

Here $\nu_{1} \leq \nu_{2} \leq \cdots \leq \nu_{r}$ denote real zeroes and $\zeta_{j}, \operatorname{Im} \zeta_{j}>0, \bar{\zeta}_{j}, 1 \leq j \leq s$, denote the others. Since the case of multiple zeroes can be considered as limit case, for the sake of simplicity, we take the assumption that they are all distinct.

Definition 3.1. For a polynomial $\Gamma(z)$, (3.7), we put the set of zeroes as follows

$$
Z(\Gamma)=Z_{\mathrm{Re}}(\Gamma) \cup Z_{\mathrm{Im}}(\Gamma) \cup \overline{Z_{\mathrm{Im}}(\Gamma)}
$$

where

$$
\begin{aligned}
& Z_{\mathrm{Re}}(\Gamma)=\left\{\nu_{i} \mid \text { real, } 1 \leq i \leq r\right\}, \\
& Z_{\mathrm{Im}}(\Gamma)=\left\{\zeta_{j} \mid \operatorname{Im} \zeta_{j}>0,1 \leq j \leq s\right\}, \\
& \bar{Z}_{\mathrm{Im}}(\Gamma)=\left\{\bar{\zeta}_{j} \mid \operatorname{Im} \bar{\zeta}_{j}<0,1 \leq j \leq s\right\} .
\end{aligned}
$$

Lemma 3.2. Let us take a polynomial $\Gamma(z),(3.7)$, and a non zero real constant c. For any $x \in \bigcup_{k=1}^{N}\left[\lambda_{2 k-1}, \lambda_{2 k}\right]$, we denote by $\sqrt{P(x)^{2}-4 A^{2}}$ the value

$$
\lim _{\varepsilon \downarrow 0} \sqrt{P(x+i \varepsilon)^{2}-4 A^{2}}, \quad x+i \varepsilon \in \mathscr{R}_{-} .
$$


Then if

$$
0 \leq \frac{c}{2 \pi i} \frac{\sqrt{P(x)^{2}-4 A^{2}}}{\Gamma(x)} \leq+\infty, \quad x \in \bigcup_{k=1}^{N}\left[\lambda_{2 k-1}, \lambda_{2 k}\right]
$$

holds, we have

1) $\nu_{i} \in Z_{\mathrm{Re}}(\Gamma)$ cannot belong to the interior of any stable band.

2) At least one of the elements $\nu_{i} \in Z_{\mathrm{Re}}(\Gamma)$ must belong to each finite unstable band. Especially $r \geq N-1$.

Proof. If we note that the sign of $\sqrt{P(x)^{2}-4 A^{2}}$ changes on the real axis as follows

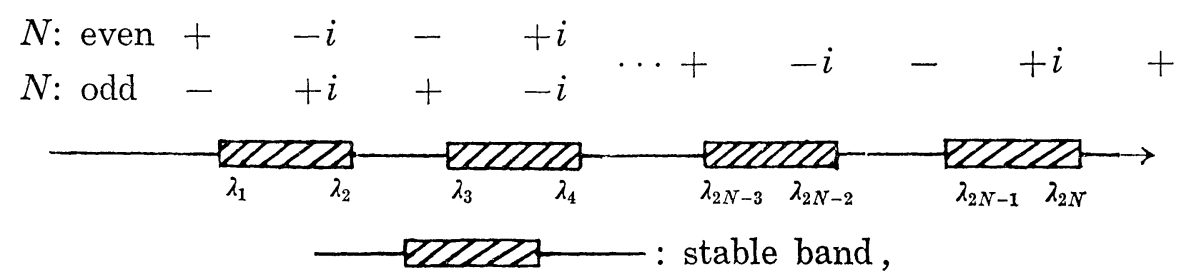

these statements are bvoious.

Remark 3.3. Under the same assumptions as in Lemma 3.2, if the integrals are finite

$$
\sum_{k=1}^{N} \lim _{\varepsilon ! 0} \frac{c}{2 \pi i} \int_{\lambda_{2 k-1+\varepsilon}}^{\lambda_{2 k}-\varepsilon} \frac{\sqrt{P(x)^{2}-4 A^{2}}}{\Gamma(x)} d x<+\infty,
$$

the form

$$
\omega=\frac{c}{2 \pi i} \frac{\sqrt{P(x)^{2}-4 A^{2}}}{\Gamma(x)} d x
$$

gives a positive Radon measure on each stable band $\left[\lambda_{2 k-1}, \lambda_{2 k}\right], 1 \leq k \leq N$.

Definition 3.3. For a pair $c, \Gamma(z)$, we put

$$
\begin{aligned}
& \text { 1) } \gamma_{i}(c, \Gamma)=-c \frac{\sqrt{P\left(\nu_{i}^{-}\right)^{2}-4 A^{2}}}{\prod_{k \neq i}\left(\nu_{i}-\nu_{k}\right) \prod_{j=1}^{s}\left(\nu_{i}-\zeta_{j}\right) \prod_{j=1}^{s}\left(\nu_{i}-\bar{\zeta}_{j}\right)}, \\
& \nu_{i} \in Z_{\mathrm{Re}}(\Gamma), \\
& \text { 2) } \mathscr{S}(c, \Gamma)=\left\{i \mid \gamma_{i}(c, \Gamma)>0\right\} .
\end{aligned}
$$

Example 3.4. Let $N=3$ and $\operatorname{deg} \Gamma(z)=6$. And the pair $c \neq 0, \Gamma(z)$ satisfy the condition in Lemma 3.2. Then the following cases occur. Here the circles colored black represent the points $\nu_{i}, i \in \mathscr{S}(c, \Gamma)$. 
I) $c>0$.

1) $r=6$.
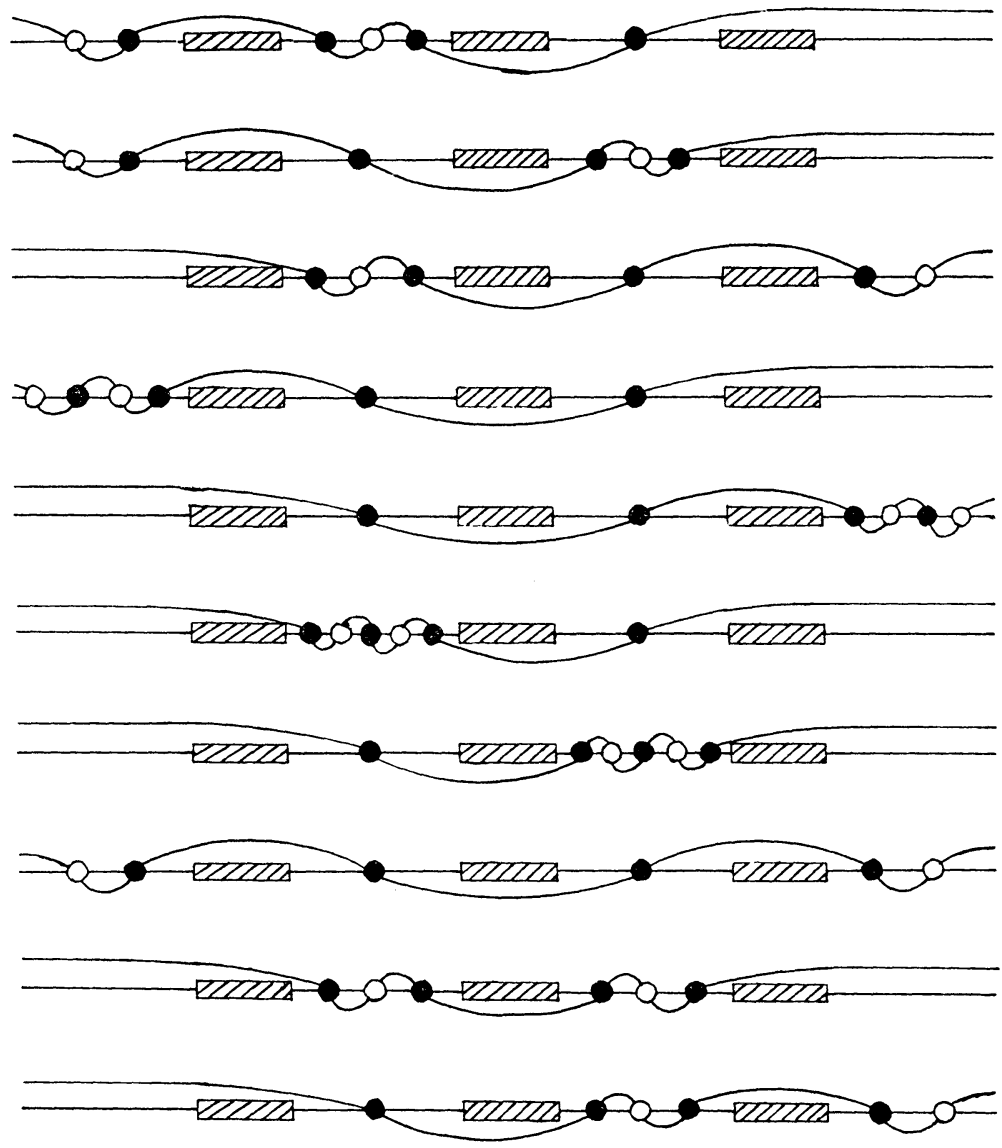

2) $r=4$.
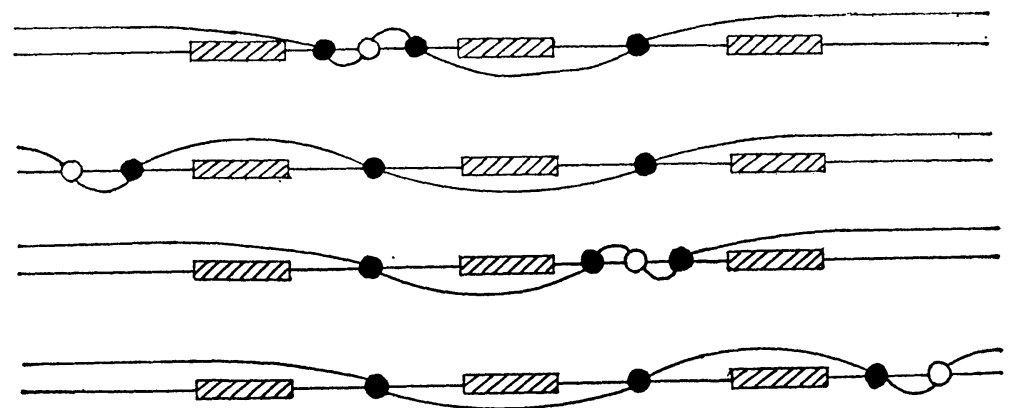
3) $r=2$.

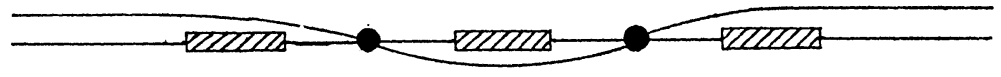

II) $c<0$.

1) $r=6$.
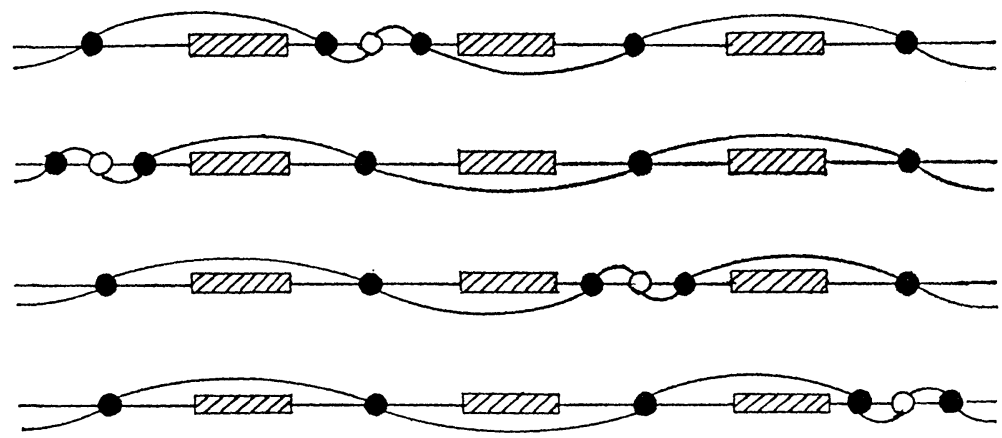

2) $r=4$.

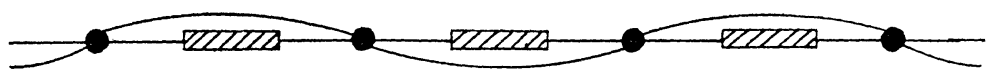

LEMMA 3.3. Let $\Phi(z), \Psi(z)$ be meromorphic functions on $\mathscr{R}$ which satisfy the relation

$$
\Psi(z)=\frac{B^{2}}{z-A-\Phi(z)}, \quad-\infty<A<+\infty, \quad 0<B
$$

Then we have

1) Let $P$ and $Q$ be zeroes of $\Psi(z)$ of first order. Then $\Psi(z)$ has $l+2$ poles in $\mathscr{R}-\{P, Q\}$ if and only if $\Phi(z)$ has $l$ poles in $\mathscr{R}-\{P, Q\}$.

2) Let $Q$ be a zero of $\Psi(z)$ of first order and $P$ be neither a zero nor a pole of it. Then $\Psi(z)$ has $l+1$ poles in $\mathscr{R}-\{P, Q\}$ if and only if $\Phi(z)$ has $l$ poles in $\mathscr{R}-\{P, Q\}$.

Proof. First let us prove 1). From (3.11), besides at the two points $P$ and $Q, \Psi(z)$ takes zeroes at the points in $\mathscr{R}-\{P, Q\}$ where $\Phi(z)$ takes poles and vice versa. Therefore if $\Phi(z)$ has $l$ poles in $\mathscr{R}-\{P, Q\}, \Psi(z)$ has $l+2$ zeroes. Hence $\Psi(z)$ must have $l+2$ poles being contained in $\mathscr{R}-\{P, Q\}$. We obtain the proof of 1 ). We can prove 2) similarly. 
Theorem 3.4. Up to a nonzero scalar factor, the conditions I. $1 . M)+$ $2 . M \alpha),($ resp. $+2 . M \beta))$ and II. $1 . M)+3 . M)+4 . M)+2 . M \alpha),($ resp. $+2 . M \beta))$, $1 \leq M<+\infty$, are equivalent, where the conditions I and II are

I. 1.M) $\psi_{M}(z)$ can be expanded into a mixed $N$ periodic $J$ fraction as follows

$$
\begin{aligned}
\psi_{M}(z)= & \frac{B_{M}^{2} \mid}{\mid z-A_{M-1}}-\frac{B_{M-1}^{2} \mid}{\mid z-A_{M-2}}-\cdots-\frac{B_{1}^{2} \mid}{\mid z-A_{0}}-\frac{B_{0}^{2}}{\mid z-a_{1}} \\
& -\frac{b_{1}^{2}}{\mid z-a_{2}}-\frac{b_{2}^{2} \mid}{\mid z-a_{3}}-\cdots
\end{aligned}
$$

where $a_{i+N}=a_{i}, b_{i+N}=b_{i}, 1 \leq i<+\infty$.

2.M $M) \quad B_{0}=b_{0}$ but $A_{0} \neq a_{N}$.

2.M $\beta) \quad B_{0} \neq b_{0}$.

II. 1.M) $\psi_{M}(z)$ can be described as follows

$$
\psi_{M}(z)=\frac{c_{M}}{2} \frac{\Lambda_{M}(z)-\frac{\sqrt{P(z)^{2}}-\overline{4 A^{2}}}{\Gamma_{M}(z)}}{}
$$

where $c_{M}$ is a non zero real number and both $\Gamma_{M}(z)$ and $\Lambda_{M}(z)$ are polynomials with real coeficients. Further $\Gamma_{M}(z)$ is monic.

2.M $\alpha) \quad\left\{\begin{array}{l}\operatorname{deg} \Gamma_{1}(z)=N, \\ \operatorname{deg} \Lambda_{1}(z)=N \text { and } \Lambda_{1}(z) \text { is monic. }\end{array}\right.$

$$
\left\{\begin{array}{l}
\operatorname{deg} \Gamma_{M}(z)=2 M+N-2, \\
\operatorname{deg} \Lambda_{M}(z)=2 M+N-3,2 \leq M<+\infty .
\end{array}\right.
$$

2.M $\beta) \quad\left\{\begin{array}{l}\operatorname{deg} \Gamma_{M}(z)=2 M+N-1, \\ \operatorname{deg} \Lambda_{M}(z)=2 M+N-2,1 \leq M<+\infty .\end{array}\right.$

3.M) The form

$$
\omega=\frac{c_{M}}{2 \pi i} \frac{\sqrt{P(x)^{2}-4 A^{2}}}{\Gamma_{M}(x)} d x
$$

gives a positive measure on each stable band $\left[\lambda_{2 k-1}, \lambda_{2 k}\right], 1 \leq$ $k \leq N$.

4.M) If we decompose

$$
\Gamma_{M}(z)=\prod_{i=1}^{r_{M}}\left(z-\nu_{M, i}\right) \prod_{j=1}^{s_{M}}\left(z-\zeta_{M, j}\right) \prod_{j=1}^{s_{M}}\left(z-\zeta_{M, j}\right)
$$

then for some subset $S \subset \mathscr{S}\left(c_{M}, \Gamma_{M}\right) \subset\left\{1,2, \cdots, r_{M}\right\}$, we have 
$(3.16)_{M}$

$$
\begin{aligned}
& A_{M}\left(\nu_{M L}\right)= \begin{cases}\left.-\sqrt{P\left(\nu_{M}, i\right.}\right)^{2}-4 A^{2} & \text { if } i \in S \\
\left.+\sqrt{P\left(\nu_{M}, i\right.}\right)^{2}-4 A^{2} & \text { if } i \in S^{c}=\left\{1,2, \cdots, r_{M}\right\}-S\end{cases} \\
& \text { and }
\end{aligned}
$$

$$
\begin{array}{ll}
\Lambda_{M}\left(\zeta_{M, j}\right)=\sqrt{P\left(\zeta_{M, j}\right)^{2}-4 A^{2}} & \text { for } \zeta_{M, j} \in Z_{\mathrm{Im}}\left(\Gamma_{M}\right), \\
\Lambda_{M}\left(\bar{\zeta}_{M, j}\right)=\overline{\Lambda_{M}\left(\zeta_{M, j}\right)}=\sqrt{P\left(\bar{\zeta}_{M, j}\right)^{2}-4 A^{2}} & \text { for } \bar{\zeta}_{M, j} \in Z_{\mathrm{Im}}\left(\Gamma_{M}\right) .
\end{array}
$$

Remark 3.5. From II. 2M), (3.16) $)_{M}$ and $(3.17)_{M}, \Lambda_{M}(z)$ is uniquely determined by Lagrange's interpolation. Therefore if we fix the pair $c_{M}$, $\Gamma_{M}(z)$, there still exist $2^{x}$, where $\chi$ is the number of the elements in $\mathscr{S}\left(c_{M}, \Gamma_{M}\right)$, ambiguities to fix $\Gamma_{M}(z)$. Indeed if we know the $J$ fraction expansion of $\psi_{M}(z),(3.12)_{M}$, which corresponds to $S$, we can calculate all the other expansions. See Appendix.

Proof. Let us divide the proof from I to II into two steps.

Step 1. We prove the following assertions.

$(*)_{M}^{\prime}:$ I. $\left.\left.1 . M\right), 2 . M\right) \Longrightarrow$ II. $\left.\left.1 . M\right), 2 . M\right)$,

$(*)_{M}^{\prime \prime}$ : I. $\left.\left.1 . M\right), 2 . M\right) \Longrightarrow \Gamma_{M L}(z)$ divides the polynomial

$$
\Lambda_{M}(z)^{2}-P(z)^{2}+4 A^{2}
$$

by induction on $M$. Let $M=1$. Then we have

$$
\psi_{1}(z)=\frac{B_{1}^{2}}{z-A_{0}-\left(\frac{B_{0}}{b_{0}}\right)^{2} \varphi(z)}
$$

$$
=\frac{B_{1}^{2}}{z-A_{0}-\frac{1}{2}\left(\frac{B_{0}}{b_{0}}\right)^{2} \cdot \frac{A_{0}(z)-\sqrt{P(z)^{2}-4 A^{2}}}{\Gamma_{0}(z)}}
$$

$$
=\frac{B_{1}^{2} \Gamma_{0}(z)\left\{\left(z-A_{0}\right) \Gamma_{0}(z)-\frac{1}{2}\left(B_{0} / b_{0}\right)^{2} \Lambda_{0}(z)-\frac{1}{2}\left(B_{0} / b_{0}\right)^{2} \sqrt{\left.P(z)^{2}-4 A^{2}\right\}}\right.}{\left(z-A_{0}\right)^{2} \Gamma_{0}(z)^{2}-\left(B_{0} / b_{0}\right)^{2}\left(z-A_{0}\right) \Gamma_{0}(z) \Lambda_{0}(z)+\frac{1}{4}\left(B_{0} / b_{0}\right)^{2}\left(\Lambda_{0}(z)^{2}-P(z)^{2}+4 A^{2}\right)} .
$$

By virtue of Corollary $2.5,(2.5)$ and $(2.21)$, we can decompose as

$$
\begin{gathered}
\Lambda_{0}(z)^{2}-P(z)^{2}+4 A^{2}=\Gamma_{0}(z) \Gamma_{0}^{c}(z), \\
\operatorname{deg} \Gamma_{0}^{c}(z) \leq N-1 .
\end{gathered}
$$

Therefore we obtain the expression

$$
\psi_{1}(z)=\frac{c_{1}^{\prime}}{2} \frac{\Lambda_{1}(z)-\frac{\sqrt{P(z)^{2}}-\overline{4 A^{2}}}{\Gamma_{1}^{\prime}(z)}}{2}
$$


where we put

$$
\begin{aligned}
c_{1}^{\prime}=\left(\frac{B_{0} B_{1}}{b_{0}}\right)^{2} & \\
\Gamma_{1}^{\prime}(z) & =\left(z-A_{0}\right)^{2} \Gamma_{0}(z)-\left(\frac{B_{0}}{b_{0}}\right)^{2}\left(z-A_{0}\right) \Lambda_{0}(z)+\frac{1}{4}\left(\frac{B_{0}}{b_{0}}\right)^{2} \Gamma_{0}^{c}(z) \\
& =\frac{b_{0}^{2}-B_{0}^{2}}{b_{0}^{2}} z^{v+1}+\{\text { terms of lower degree }\}, \\
\Lambda_{1}(z) & =2\left(\frac{b_{0}}{B_{0}}\right)^{2}\left(z-A_{0}\right) \Gamma_{0}(z)-\Lambda_{0}(z) \\
& =\frac{2 b_{0}^{2}-B_{0}^{2}}{B_{0}^{2}} z^{v}+\{\text { terms of lower degree }\} .
\end{aligned}
$$

According to $B_{0}=b_{0}$ or $B_{0} \neq b_{0}$ we put

$$
\left\{\begin{array}{l}
c_{1}=\frac{c_{1}^{\prime}}{d_{1}} \\
\Gamma_{1}(z)=\frac{1}{d_{1}} \Gamma_{1}^{\prime}(z),
\end{array}\right.
$$

where $d_{1}$ is the coefficient of $z^{v}$ in $\Gamma_{1}^{\prime}(z)$, and

$$
\left\{\begin{array}{l}
c_{1}=c_{1}^{\prime} \frac{b_{0}^{2}}{b_{0}^{2}-B_{0}^{2}}=\frac{B_{0}^{2} B_{1}^{2}}{b_{0}^{2}-B_{0}^{2}} \\
\Gamma_{1}(z)=\frac{b_{0}^{2}}{b_{0}^{2}-B_{0}^{2}} \Gamma_{1}^{\prime}(z),
\end{array}\right.
$$

we get the assertion $(*)_{1}^{\prime}$. Notice that if $B_{0}=b_{0}$ and $d_{1}=0$ then it is the special case $A_{0}=a_{s}$ being excluded. Next let us prove $(*)_{1}^{\prime \prime}$. Let $B_{0} \neq b_{0}$. Then if we use Lemma 3.3. 1), we can show that $\psi_{1}(z)$ must have $N+1$ poles in $\mathscr{R}-\{P, Q\}$. But from the expression (3.13) , for any zero $z_{0} \in$ $Z\left(\Gamma_{1}\right)$, at least one of the two lifted points $z_{0}^{+}, z_{0}^{-}$has to become a pole of $\psi_{1}(z)$. Since the degree of $\Gamma_{1}(z)$ is equal to $N+1$, this means that

$$
A_{1}\left(z_{0}\right)=\sqrt{P\left(z_{0}^{-}\right)^{2}-\overline{4 A^{2}}},
$$

or

$$
A_{1}\left(z_{0}\right)=\sqrt{ } P\left(z_{0}^{+}\right)^{2}-4 A^{2}=-\sqrt{ } P\left(z_{0}^{-}\right)^{2}-\overline{4 A^{2}}
$$

holds. Hence $(*)_{1}^{\prime \prime}$ follows. In case $B_{0}=b_{0}$ but $A_{0} \neq a_{N}$, Lemma 3.3. 2$)$ is applicable and we can prove $(*)_{1}^{\prime \prime}$ similarly.

By use of induction, since we can rewrite 


$$
\begin{aligned}
& \psi_{Y+1}(z)=\frac{B_{M+1}^{2}}{z-A_{M}-\psi_{M}(z)} \\
& =\frac{B_{M+1}^{2}}{z-A_{M}-\frac{c_{M} A_{M H}(z)-\sqrt{ } P(z)^{2}-4 A^{2}}{2}} \\
& B_{M+1}^{2} \Gamma_{M}(z)\left\{\left(z-A_{M}\right) \Gamma_{M}(z)-\frac{c_{M}}{2} \Lambda_{M}(\mathrm{z})-\frac{c_{M}}{2} \cdot \sqrt{ } P(z)^{-}-4 A^{2}\right\} \\
& \left(z-A_{M}\right)^{2} \Gamma_{M}(z)^{2}-c_{M}\left(z-A_{M}\right) \Gamma_{M}(z) \Lambda_{M}(z)+\left(\frac{c_{M}}{2}\right)^{2}\left(\Lambda_{M}(z)^{-}-P(z)^{2}+4 A^{2}\right),
\end{aligned}
$$

the rest we must do is to show that $(*)_{M}^{\prime \prime}$ follows from $(*)_{Y}^{\prime}$ and $(*)_{M-1}^{\prime \prime}$. For $M \geq 2$, we can write

$$
\begin{aligned}
\psi_{M}(z) & =\frac{B_{M}^{2}}{z-A_{M}-\psi_{M-1}(z)} \\
& =\frac{c_{M} A_{Y}(z)-\sqrt{P(z)^{2}}-4 A^{2}}{2},
\end{aligned}
$$

and use Lemma 3.3. 1). The number of poles in $\mathscr{R}-\{P, Q\}$ of $\psi_{y}(z)$ is equal to

$$
\begin{aligned}
& 2 M+N-1 \text { if } B_{0}=b_{0}, \\
& 2 M+N-2 \text { if } B_{0}=b_{0} \text { but } A_{0} \neq a_{.},
\end{aligned}
$$

and it coincides with the degree of $\Gamma_{M}(z)$. But (3.20) implies that for any zero $z_{0} \in Z\left(\Gamma_{y}\right)$, at least one of the two lifted points $z_{i j}^{-}, z_{0}^{-}$must be a pole of $\psi_{y}(z)$. Therefore

$$
\Lambda_{M}\left(z_{0}\right)=\sqrt{P\left(z_{0}^{-}\right)^{2}-4 A^{2}}
$$

or

$$
=\sqrt{ } P\left(z_{0}^{+}\right)^{2}-4 A^{2}=-\sqrt{ } P\left(z_{0}^{-}\right)^{2}-4 A^{2}
$$

holds and we obtain the assertion $(*)^{\prime \prime}$.

Step 2. Let us prove $3 . M$ ) and $4 . M)$. Since $\psi_{y M}(z)$ admits the $J$ fraction expansion, $(3.12)_{M}$, it is expressible in a Stieltjes transform

$$
\psi_{M}(z)=\int_{-\infty}^{+\infty} d \mu(x)
$$

for some Stieltjes measure $d_{\mu}(x)$. In particular, $\psi_{y}(z)$ is holomorphic in the domain $\mathscr{R}_{-}$- -real axis\}, so $(3.17)_{I I}$ must be satisfied. 
Lemma 3.6. Any real zero $\nu_{M, i} \in Z_{\mathrm{Re}}\left(\Gamma_{M}\right)$ cannot belong to the interior of stable bands $\left[\lambda_{2 k-1}, \lambda_{2 k}\right], 1 \leq k \leq N$.

Proof of Lemma 3.6. If $\nu_{M, i}$ lies there, it follows that

$$
\left.\Lambda_{M}\left(\nu_{M, i}\right)=\sqrt{P\left(\nu_{M}^{-}, i\right.}\right)^{2}-\overline{4 A^{2}}
$$

or

$$
\left.-\sqrt{P\left(\nu_{M}, i\right.}\right)^{2}-4 A^{2} .
$$

Then the left hand side is real but the right hand sides are both pure imaginary This is a contradiction.

Let $C$ and $C_{k}, 1 \leq k \leq N$, be anti-clockwise contours in $\mathscr{R}_{\text {_ }}$ as below

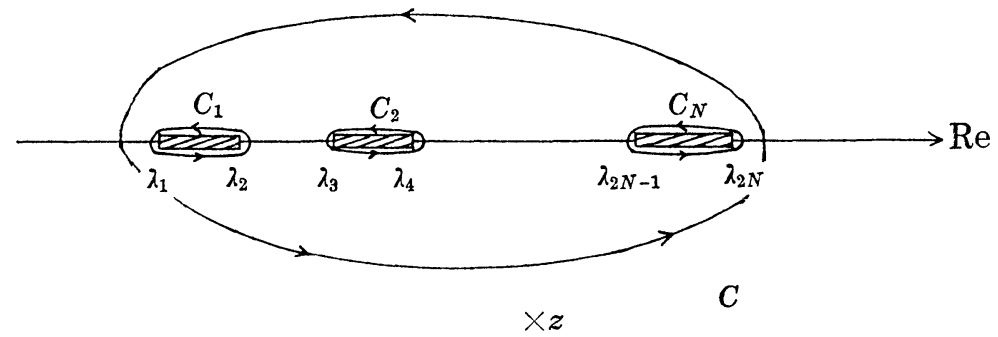

where the contour $C$ contains all stable bands and all zeroes of $\Gamma_{y}(z)$ inside. Then for any element $z$ in the outside of $C$, we can write

$$
\begin{aligned}
\psi_{M}(z) & =\frac{1}{2 \pi i}-\oint_{C} \frac{\psi_{M}(x)}{z-x} d x \\
& =\sum_{j=1}^{r_{M}} \frac{\gamma_{M, j}}{z-\nu_{M, j}}+\frac{1}{2 \pi i} \sum_{j=1}^{N} \oint_{C_{j}} \frac{\psi_{M}(x)}{z-x} d x \\
& =\sum_{j=1}^{r_{M}} \frac{\gamma_{M, j}}{z-\nu_{M, j}}+\frac{c_{M}}{2 \pi i} \sum_{j=1}^{N} \int_{\lambda_{2 j-1}}^{\lambda_{2 j}} \frac{\sqrt{P(x)^{2}-4 A^{2}}}{(z-x) \Gamma_{M}(x)} d x
\end{aligned}
$$

where

$$
\begin{aligned}
\gamma_{M, j} & =\operatorname{Res}_{\nu_{M, j}} \psi_{M}(z) \\
& =\left\{\begin{array}{cl}
\gamma_{j}\left(c_{M}, \Gamma_{M}\right) & \text { if } \Lambda_{M}\left(\nu_{M, j}\right)=-\sqrt{P\left(\nu_{M}^{-}, j\right)^{2}-4 A^{2}} \\
0 & \text { if } \left.\Lambda_{M}\left(\nu_{M, j}\right)=+\sqrt{P\left(\nu_{M}^{-}, j\right.}\right)^{2}-4 A^{2}
\end{array}\right.
\end{aligned}
$$

And we put

$$
S=\left\{j \mid \Lambda_{M}\left(\nu_{M, j}\right)=-\sqrt{P\left(\nu_{M}^{-}, j\right)^{2}-4 A^{2}}\right\} .
$$

Since the Stieltjes measure $d \mu(x)$ is uniquely determined, we have 


$$
d \mu(x)=d \mu_{d}(x)+d \mu_{c}(x)
$$

where we put

$$
\begin{aligned}
& d \mu_{d}(x)=\sum_{j \in S} \gamma_{M, j} \cdot \delta\left(x-\nu_{M, j}\right) d x, \quad \text { discrete measure, } \\
& d \mu_{c}(x)=\sum_{j=1}^{N} \frac{c_{M}}{2 \pi i} \chi_{\left[\lambda_{2 j-1}, i_{2}\right]}(x) \frac{\sqrt{P(x)^{2}-4 A^{2}}}{\Gamma_{M}(x)} d x, \\
& \text { continuous measure. }
\end{aligned}
$$

Then the positivity of $d \mu(x)$ implies II. 3.M) and 4.M).

Now let us prove the converse II to I. If we assume II then $\psi_{M I}(z)$ can be expressed in the Stieltjes transform of the measure defined by (3.27) for some subset $S \subset \mathscr{S}\left(c_{y}, \Gamma_{y}\right)$. Therefore it admits a $J$ fraction expansion

$$
\begin{aligned}
y_{M}(z)= & \frac{c_{M}}{2} \frac{A_{M}(z)-\sqrt{P(z)^{2}-4 A^{2}}}{\Gamma_{M}(z)} \\
= & \frac{B_{M}^{2}}{z-A_{M-1}}-\frac{B_{M-1}^{2}}{z-A_{M-2}}-\cdots-\frac{B_{1}^{2}}{z-A_{0}} \\
& -\frac{B_{0}^{2}}{z-a_{1}}-\frac{b_{1}^{2}}{z-a_{2}}-\cdots
\end{aligned}
$$

At this stage, we do not know the periodicity. But since we can decompose as

$$
\begin{aligned}
& \frac{c_{M}}{2}\left(A_{M}(z)^{2}-P(z)^{2}+4 A^{2}\right)=\frac{2}{c_{M-1}^{\prime}} \Gamma_{M}(z) \cdot \Gamma_{M-1}(z), \\
& \Gamma_{y-1}(z) \text { is monic, }
\end{aligned}
$$

we have

$$
\begin{aligned}
& y_{M}(z)= \frac{1}{\Gamma_{M}(z)\left(\Lambda_{M}(z)+\sqrt{ } P(z)^{2}-4 A^{2}\right)} \\
& \frac{c_{M}}{2}\left(A_{M}(z)^{2}-P(z)^{2}+4 A^{2}\right) \\
&=\frac{1}{a z+b-\frac{c_{Y-1}^{\prime}}{2} \frac{A_{M-1}(z)-\sqrt{ } P(z)^{2}-4 A^{2}}{\Gamma_{M-1}(z)}}
\end{aligned}
$$

Here we draw the linear part $a z+b$ so that $\operatorname{deg} A_{M-1}(z)$ satisfies the condition 2.M-1). If we compare (3.28) and (3.30), we have 


$$
B_{M}=\frac{1}{\sqrt{ } a}, \quad A_{M-1}=-\frac{b}{a}, \quad c_{M-1}=\frac{c_{M-1}^{\prime}}{a}
$$

and

$$
\begin{aligned}
\frac{c_{M-1}}{2} & \frac{\Lambda_{M-1}(z)-\sqrt{P(z)^{2}-4 A^{2}}}{\Gamma_{M-1}(z)} \\
= & \frac{B_{M-1}^{2} \mid}{z-A_{M-2}}-\frac{B_{M-2}^{2}}{z-A_{M-3}}-\cdots-\frac{B_{1}^{2}}{z-A_{0}} \\
& -\frac{B_{0}^{2}}{z-a_{1}}-\frac{b_{1}^{2}}{z-a_{2}}-\cdots
\end{aligned}
$$

If we use Lemma 3.3 and note the $J$ fraction expansion (3.31), the condition $3 . M-1$ ) and $4 . M-1$ ) are also satisfied for this function. Since the conditions of the case $M=0$ coincide with those of purely periodic $J$ fractions, we can obtain the proof from II to I by induction on $M$.

We have already given the proof of the following theorem.

THEOREM 3.7. Let $\psi_{M}(z)=\psi_{M, S}(z)$ be a mixed $N$ periodic $J$ fraction (3.12) $)_{M}$ in Theorem 3.4 corresponding to some subset $S \subset \mathscr{S}\left(c_{M}, \Gamma_{M}\right)$. Then

1) $\psi_{M}, s(z)$ can be described in the Stieltjes transform of the measure

$$
d_{\mu_{S}}(x)=d \mu_{S, d}(x)+d \mu_{c}(x)
$$

where

$$
\begin{aligned}
& d \mu_{S, d}(x)=\sum_{j \in S} \gamma_{M, j} \delta\left(x-\nu_{M, j}\right) d x, \\
& d \mu_{c}(x)=\sum_{j=1}^{N} \frac{c_{M}}{2 \pi i} \chi_{\left[\lambda_{2 j-1}, \lambda_{2} j\right]}(x) \frac{\sqrt{P(x)^{2}-}-\overline{4} \bar{A}^{2}}{\Gamma_{M}(x)} d x .
\end{aligned}
$$

2) For any two subsets $S, S^{\prime} \subset \mathscr{S}\left(c_{M}, \Gamma_{M}\right)$, we have the relation as follows

$$
\psi_{M, S}(z)=\psi_{M, S}(z)+\sum_{j \in S^{\prime}-S} \frac{\gamma_{M, j}}{z-\nu_{M, j}}-\sum_{k \in S-S^{\prime}} \frac{\gamma_{M, k}}{z-\nu_{M, h}} .
$$

\section{§4. Appendix}

We take an arbitrary $J$ fraction $\varphi(z)$ and expand it at the infinity

$$
\begin{aligned}
\varphi(z) & =\frac{b_{0}^{2}}{z-a_{1}}-\frac{b_{1}^{2}}{z-a_{2}}-\frac{b_{2}^{2}}{z-a_{3}}-\cdots \\
& =\frac{c_{0}}{z^{1}}+\frac{c_{1}}{z^{2}}+\frac{c_{2}}{z^{3}}+\cdots .
\end{aligned}
$$


Then the coefficients $a_{\imath}, b_{i}$ are calculated from the moments $c_{l}$ by the following formulae

$$
\begin{aligned}
a_{n} & =\frac{1}{H_{n-2}^{\prime}}\left(\frac{H_{n-2} H_{n-1}^{\prime}}{H_{n-1}}+\frac{H_{n-1} H_{n-3}^{\prime}}{H_{n-2}}\right), \\
b_{n}^{2} & =\frac{H_{n} H_{n-2}}{H_{n-1}^{2}},
\end{aligned}
$$

with

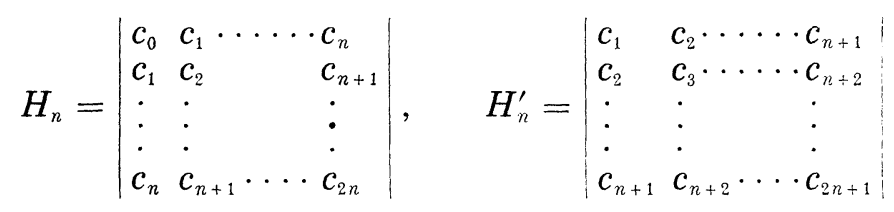

and by definition $H_{-2}^{\prime}=0, H_{-1}^{\prime}=H_{-1}=1$.

If we expand the right hand side of (3.32) at the infinity and use these formulae, we can calculate the $J$ fraction expansion of $\psi_{M, S^{\prime}}(z)$ from that of $\psi_{M, S}(z)$ at finite steps.

\section{REFERENCES}

[1] Jones, W. B. and Thron, W. J., Continued Fractıons, Addison-Wesley, 1980.

[2] Kato, Y., On the spectral density of periodic Jacobi matrices, Proceedings of RIMS Symposium on Non-Linear Integrable Systems-Classical Theory and Quantum Theory, Kyoto Japan, 1981, 153-181, World Science Publishing Co., 1983.

[3] - Periodic Jacobi continued fractions, International Symposium on Orthogonal Polynomials and their Applications, Bar-le-Duc France, 1984, Springer Lecture Notes in Math.

[4] Lambin, Ph. and Gaspard, J.-P., Continued fraction technique for tight-binding. systems, A generalized-moments method, Phys. Rev. B, 26 (1982), 4356-4368.

[5] Magnus, A., Recurrence coefficients for orthogonal polynomials on connected and non connected sets, Springer Lecture Notes in Math., 765 (1979), 150-171.

[ 6 ] van Moerbeke, P., The spectrum of Jacobi matrices, Invent. Math., 37 (1976), 45-81.

[ 7] - and Mumford, D., The spectrum of difference operators and algebraic curves, Acta Math., 143 (1979), 93-154.

[8] Moser, J., Finitely many mass points on the line under the influence of an exponential potential-an integrable system, in Battelle Recontres Summer Lectures, Springer Lecture Notes in Phys., 1974, 467-497.

[9] Nuttall, J. and Singh, S. R., Orthogonal polynomials and Padé approximants associated with a system of arcs, J. Approx. Theory, 21 (1977), 1-42.

[10] Szegö, G., Orthogonal Polynomials, A.M.S. Colloq. XXIII, 1939.

[11] Turchi, P., Ducastelle, F. and Treglia, G., Band gaps and asymptotic behaviour of continued fraction coefficients, J. Phys. C, 15 (1982), 2891-2924. 
[12] Woess, W., Random walks and periodic continued fractions, preprint, 1984, to appear in Adv. in Appl. Probab.

Department of Engineering Mathematics

Faculty of Engineering

Nagoya University

Chikusa-ku, Nagoya, 464, JAPAN 